\title{
An Ultra-Wideband Radar System for Through-the-Wall Imaging using a Mobile Robot
}

\author{
A. Judson Braga and Camillo Gentile \\ National Institute of Standards and Technology \\ Emerging and Mobile Networking Technologies Group \\ Gaithersburg, Maryland, USA
}

\begin{abstract}
High-resolution imaging through walls and other materials using microwave signals serves amongst other applications in the rapid detection of human maneuvering, rescue missions in collapsed buildings, and target feature extraction. While narrowband Doppler radar in the millimeter-wave or infrared spectrum can provide good resolution through clothing and packaging, penetration through denser sheetrock, plaster, and brick requires operation below $10 \mathrm{GHz}$; this band however yields poor resolution. As an alternative, Ultra-Wideband radar operating in this band boosts the bandwidth which translates into fine range resolution; still it requires an aperture length of several meters for comparable cross-range resolution. The associated cost and portability in realizing such an aperture through antenna arrays or fixed-length scanners have limited their lengths to the order of 1 meter in prototype systems to date. In this work, we propose a novel aperture taking form as a variable-length scanner or mobile robot. The wide dynamic range of our system coupled with its unrestricted aperture length allows us to generate high-resolution images up to a range of 8 meters or more.
\end{abstract}

Index Terms - Synthetic aperture radar

\section{INTRODUCTION}

High-resolution imaging through obstacles such as walls, doors, and other visually opaque materials using microwave signals is considered a powerful tool in both military and commercial applications including the rapid detection of human maneuvering, rescue missions in avalanches or collapsed buildings, target feature extraction, and surveillance and reconnaissance. While narrowband Doppler radar in the millimeterwave or infrared spectrum has been shown to provide good resolution through clothing and packaging [1], penetration through denser materials like fiberglass, wood, sheetrock, plaster, brick, and concrete blocks requires operation below $10 \mathrm{GHz}$ [2], [3]; this band however yields poor resolution, limiting application.

Ultra-Wideband (UWB) signals are characterized by a bandwidth greater than $500 \mathrm{MHz}$ or one exceeding $20 \%$ the center frequency of radiation [4]. The advent of practical high-speed sampling and pulse generation in recent years, coupled with the approval of the FCC unlicensed band from $3.1-10.6 \mathrm{GHz}$ in 2002, has fed the commercial success of UWB technology in fast-rate communications and precision ranging. This success has however obscured its potential as an alternative to Doppler radar for through-the-wall imaging: UWB compensates for the significant drop in center frequency by boosting the bandwidth which translates into fine range resolution. UWB also offers immunity to interference and low probability of interception, of particular interest in military applications.
Prototype UWB radar systems [5], [6], [7], [8], [9], [10] employing bandwidths from $0.5 \mathrm{GHz}$ up to $6.4 \mathrm{GHz}$ can achieve fine spatial resolution, but the cost and portability of the antenna arrays or fixed-length scanners in realizing the synthetic aperture limit its length to the order of 1 meter. This in turn limits the range to the same order, making the systems more suitable for target feature extraction in groundpenetrating radar or terrain profiling [11], [12], [13]; otherwise if geared towards applications like target identification through foliage must sacrifice cross-range resolution for longer range [1], [14]. An UWB radar system for through-the-wall imaging with both long range and fine resolution requires an aperture length on the order of several meters [15]. In this work, we propose a novel variable-length scanner with monostatic antennas mounted on a platform with wheels, or mobile robot. The high dynamic range coupled with the unrestricted aperture length of the robot allows us to generate high-resolution images up to 8 meters or more.

The paper is organized as follows: Section II introduces the Ultra-Wideband radar model together with the spatial and frequency scans used for measurement. The subsequent section details the design of the monostatic measurement system realized through a vector analyzer coupled to a mobile robot, followed in Section IV by a migration procedure to transform the measurements into a two-dimensional image. The results Section $\mathrm{V}$ shows a number of images generated in different environments, and the last section summarizes our work.

\section{ULTRA-WIDEBAND RADAR}

A radar image of the objects in an environment can be generated by transmitting a signal and measuring the roundtrip time of its arrivals reflected off the objects and returned to the receiver. Fig. 1(a) illustrates a spatial scan using our radar system: we sample the cross-range $x$ of aperture length $X$ with interval $\Delta x$, recording the round-trip arrival time $t$ of the closest object at each position $x_{p}, p=1 . . P, P=\frac{X}{\Delta x}+1$. As the signal travels at the speed of light $c$, the time can be halved and converted to the range

$$
z=\frac{c \cdot t}{2}
$$

The radar system effectively measures the temporal response

$$
h\left(t, x_{p}, z=0\right)=\sum_{k} a_{k} \delta\left(t-t_{k}\right)
$$




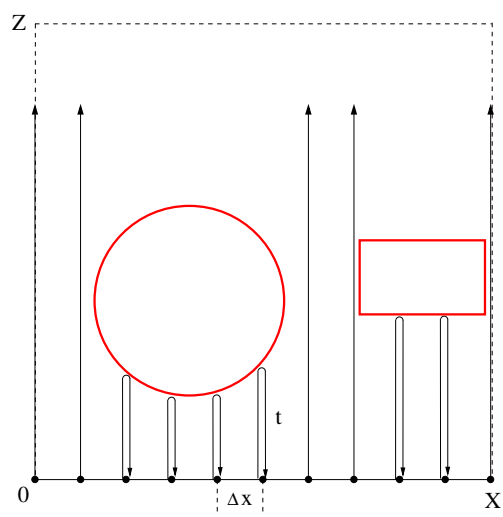

(a) Spatial scan

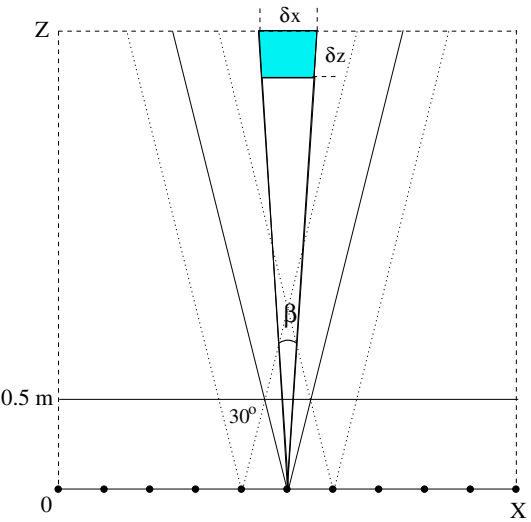

(b) Range and cross-range resolutions

Fig. 1. The Ultra-Wideband synthetic aperture radar system.

of the channel between the transmitter and receiver collocated at $\left(x_{p}, z=0\right)$. It is composed from multipath arrivals indexed through $k$, where $t_{k}$ denotes the arrival time and the complex amplitude $a_{k}$ accounts for both attenuation and phase shift in propagation [16].

The temporal response has a frequency response

$$
H\left(f, x_{p}, z=0\right)=\sum_{k} a_{k} e^{-j 2 \pi f t_{k}},
$$

suggesting that the channel can be characterized through a frequency scan: we sample $H\left(f, x_{p}, z=0\right)=\frac{Y(f)}{X(f)}$ with interval $\Delta f$ in the band of bandwidth $B$ and center frequency $f_{c}$, emitting tones $X(f)$ from the transmitter and then measuring $Y(f)$ at the receiver. Measuring the channel in the frequency domain offers the advantages of wider dynamic range, higher mean power, and lower noise figure over transmitting a UWB pulse and recording the temporal response directly [11].

\section{SYSTEM DESIGN}

\section{A. System parameters}

Range resolution $\delta z$ : The range resolution of our radar system depends on its ability to discriminate arrivals reflected from both target and surrounding objects. This is achieved when the arrivals are separated in time by at least a pulse width. From (1), a pulse of duration $\delta t$ corresponds to a range increment of $\delta z=\frac{c \cdot \delta t}{2}$. Then a transmitted spectrum $X(f)$ with unit amplitude and zero phase transforms to the time domain as a sinc pulse with width $\delta t=\frac{1}{B}$ [17]. So the range resolution of the system is $\delta z=\frac{c}{2 B}$, or $2.5 \mathrm{~cm}$ for $B=6 \mathrm{GHz}$.

Frequency sample interval $\Delta f:$ The discreteness of the spectrum $Y(f)$ means that the received sinc pulses are actually periodic with interval $T=\frac{1}{\Delta f}$ [17]. Choosing $\Delta f=1.25 \mathrm{MHz}$ allows for a maximum multipath spread $T$ of $800 \mathrm{~ns}$ which proves sufficient throughout all tested environments for the arrivals to subside and avoid time aliasing between intervals.

Cross-range resolution $\delta x$ : A synthetic aperture reduces the real beamwidth of an antenna to provide a finer cross-range resolution. The synthetic beamwidth $\beta$ of our radar system in Fig. 1(b) is equal to ${\frac{c}{2 f_{c} X}}^{1}$ [15]. For small angles as in our case, the chord length is approximately equal to $\beta \cdot z$ [15], which together with the range resolution $\delta z$ bounds the shaded resolution cell. As the chord length depends on $z$, the maximum range $Z$ sets the (worst) cross-range resolution $\delta x=\frac{c \cdot Z}{2 \cdot f_{c} \cdot X}$. In our system, we assume an aperture length equal to the maximum range in which all the reflectors lie; so for $X=Z$, the cross-range resolution depends only on the center frequency and is $3 \mathrm{~cm}$ for $f_{c}=5 \mathrm{GHz}$. Hence we have designed an UWB radar system with comparable resolution in both range and cross-range.

Cross-range sample interval $\Delta x$ : In order for the radar system to detect an object, it must lie within the real beamwidth of the antennas. As the beam extends from zero at antenna, there is little coverage at short range. The real beamwidth of our antennas is about $30^{\circ}$, so at a range of $0.5 \mathrm{~m}$ the chord length or coverage is about $0.26 \mathrm{~m}$. Then in designing a system to cover the whole scan length for ranges in excess of $0.5 \mathrm{~m}$ in reference to Fig. 1(b), the cross-range sample space $\Delta x$ should be less than $26 \mathrm{~cm}$.

\section{B. System hardware}

Fig. 2 displays the block diagram (a) and photograph (b) of our radar system. The vector network analyzer (VNA) controls the entire system, alternating between spatial and frequency scans. The Tx and Rx antennas are mounted with $47 \mathrm{~cm}$ vertical separation on a wooden post (sturdy yet minimizes reflection) attached to the base of the mobile platform; their midpoint sits at $1.5 \mathrm{~m}$ (half the average ceiling height). The RS232 serial interface of the VNA serves to reposition the robot through an $802.11 \mathrm{~b}$ wireless communication link. There is no transmission on the link during a frequency scan to avoid interference at $2.4 \mathrm{GHz}$. The two fixed axles of the robot permit only rectilinear (forward and reverse) motion with 1 $\mathrm{cm}$ step granularity and less than $0.2 \%$ tolerance in the offaxis. The coaxial cable enables $\pm 6 \mathrm{~m}$ translation of the robot from the VNA for a combined maximum aperture length of $12 \mathrm{~m}$ without displacing the latter.

${ }^{1}$ The actual synthetic beamwidth depends on the tilt of the beam not necessarily perpendicular to the scan axis as shown in the figure. 


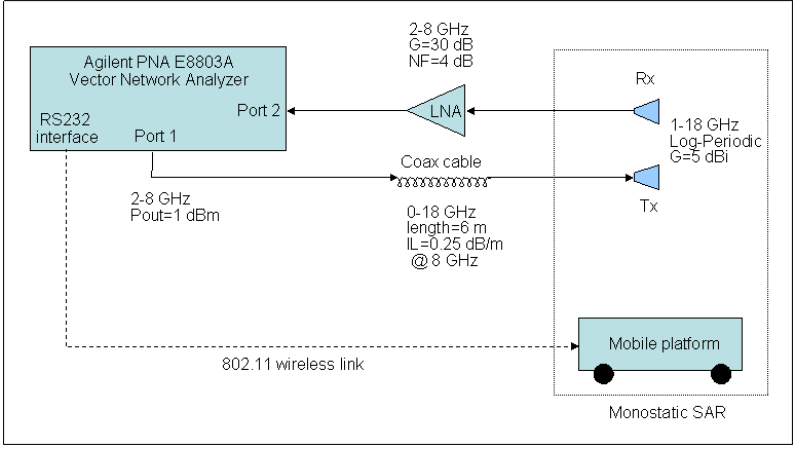

(a) Block diagram

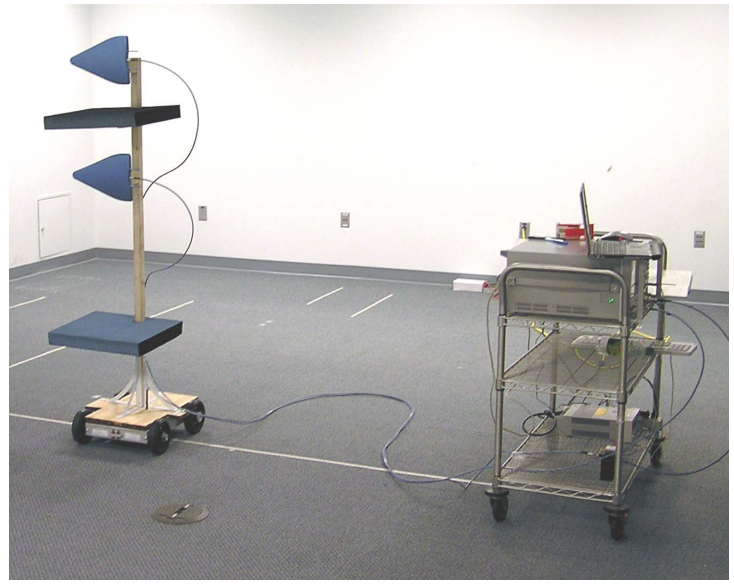

(b) Photograph

Fig. 2. The block diagram and photograph of our Ultra-Wideband synthetic aperture radar system using a mobile robot.

Most radar systems employ directional antennas as they can irradiate reflectors only in the target direction, both to maximize emission power and prevent scattering from ambient objects or walls in other directions, especially behind the robot. We chose the log-periodic antenna over the horn antenna due to its low cost, more stable radiation pattern across our band, higher gain at $8 \mathrm{GHz}$ compared to $2 \mathrm{GHz}$ to compensate for frequency fading, and lighter weight $(0.25 \mathrm{~kg})$, making it more practical for our mobile platform which supports a maximum weight of $5 \mathrm{~kg}$. While they tend to have greater time dispersity compared to the horn [11], this can be corrected through separate calibration at each frequency as explained in the sequel.

During the frequency scan, the VNA emits a series of tones with frequency $f$ at Port 1 and measures the relative amplitude and phase $S^{21}(f)$ with respect to Port 2, providing automatic phase synchronization between the two ports. The signal radiates at approximately $1 \mathrm{dBm}$ from the Tx antenna; when it returns to the receiver, the low-noise amplifier (LNA) boosts the signal above the noise floor of Port 2 before feeding it back. The absorber placed between the antennas reduces the strength of the direct-path arrival which would otherwise saturate the LNA; the absorber below the receiver antenna reduces any reflections off the floor. Transmitting at greater than $1 \mathrm{dBm}$ would also saturate the LNA for reflectors closer than $0.5 \mathrm{~m}$, so to compensate we scan at the minimum $\Delta x=$ $1 \mathrm{~cm}$ to increase the total received power summed from each scan position. The dynamic range of the system was computed as $140 \mathrm{~dB}$ according to [18] for an IF bandwidth of $1 \mathrm{kHz}$ and a SNR of $15 \mathrm{~dB}$ at the receiver. The $S_{p}^{21}(f)$-parameter of the network in Fig. 2(a) can be expressed as a product of the cable, the $T x$-antenna, the propagation channel, the $R x$-antenna, and the LNA as

$$
\begin{aligned}
S_{p}^{21}(f) & =H_{c a b}(f) \cdot H_{T x}(f) \cdot H\left(f, x_{p}, z=0\right) \cdot H_{L N A}(f) \cdot H_{R x}(f) \\
& =H_{c a b}(f) \cdot \underbrace{H_{T x}(f) \cdot H_{R x}(f)}_{H_{\text {ant }}(f)} \cdot H\left(f, x_{p}, z=0\right) \cdot H_{L N A}(f) .
\end{aligned}
$$

The frequency response $H$ is extracted by individually measuring the transmission responses $H_{c a b}, H_{a n t}$, and $H_{L N A}$ in advance and deembedding them from (4). Measuring the characteristics of the antennas on a flat open field with dimensions exceeding $100 \mathrm{~m} \times 100 \mathrm{~m}$ reduced ambient multipath to a single ground bounce which we removed by placing electromagnetic absorbers on the ground between the antennas. We pointed the antennas towards each other and separated them by a distance of $1.5 \mathrm{~m}$ to avoid the near-field effects.

\section{IMAGE GENERATION}

A procedure known as migration generates a twodimensional image from the measured frequency response in (3) through the Phase-Shift Method [19]. The latter provides more accurate results than the alternative Kirchhoff and F-K Methods and also lends most readily to varying propagation velocity $^{2}$ [20]. The first step is to convert the response into a wavefield $H^{2}\left(f, k_{x}, z=0\right)$ through the Fourier Transform from the spatial domain $x$ to the wavenumber domain $k_{x}$

$$
H^{2}\left(f, k_{x}, z=0\right)=\sum_{p=1}^{P} H\left(f, x_{p}, z=0\right) e^{-j k_{x} x_{p}} .
$$

The method proceeds by propagating the wavefield downward to $z>0$ by adding the appropriate phase shift in $z$ through the Fourier kernel

$$
H^{2}\left(f, k_{x}, z\right)=H^{2}\left(f, k_{x}, z=0\right) e^{-j k_{z} z},
$$

where $k_{z}$ denotes the wavenumber in the $z$ direction. Since the wavefield obeys the dispersion relation of the scalar wave equation [15]

$$
k_{z}\left(f, k_{x}\right)=\sqrt{\left(\frac{2 \pi f}{c}\right)^{2}-k_{x}^{2}},
$$

it serves to distinguish $H^{2}$ as a wavefield from an arbitrary function and so can be used to infer information on $z$ by

${ }^{2}$ In ground penetrating radar applications for example, the signal slows down with respect to free space as it propagates through soil. 
substitution into (6). Note that $k_{z}$ is now a function of the measured quantities $f$ and $k_{x}$ (through $x$ ).

Once the wavefield has been propagated to $z$, the complete frequency response can be recovered through the Inverse Fourier Transform of $H^{2}\left(f, k_{x}, z\right)$ from $k_{x}$ back to $x$

$$
H(f, x, z)=\Delta x \int_{0}^{\frac{1}{\Delta x}} H^{2}\left(f, k_{x}, z\right) e^{j k_{x} x} d x .
$$

Similarly the complete temporal response can be recovered from $H(f, x, z)$ as

$$
h(t, x, z)=\frac{\Delta f}{B} \sum_{l=1}^{\frac{B}{\Delta f}} H(f, x, z) e^{j 2 \pi f t},
$$

where $f=f_{c}-\frac{B}{2}+l \cdot \Delta f$.

Instead of the actual spatial scan described in Section II, consider separating the transmitter and receiver in a virtual scan in which the transmitter moves along the contours of the reflectors rather than along the cross-range. The Exploding Reflector Concept [15] maintains that the temporal response measured along the cross-range both in the actual and virtual scans is the same, the only difference being that the actual time comprises both the forward and return travel from the transmitter, off the reflector, and back to the receiver while the virtual time comprises only the return time from the reflector to the receiver. Then an image of the reflectors can be generated by examining the temporal response $h(t, x, z)$ at $t=0$ to visualize the reflectors as they "explode." To compensate for the forward travel time, it suffices to half the propagation velocity by replacing $\frac{c}{2}$ in (7). See [15] for implementation details.

\section{RESUlTS}

This section displays four images generated from measurements taken in different environments using our radar system. As explained above, the actual image is recovered from the amplitude of the impulse response viewed at $t=0$, or $|h(t=0, x, z)|$ from (9), and plotted as range $z$ versus cross-range $x$.

Fig. 3(a) shows a radar image generated from a 5-meter scan of four objects mounted on tripods and placed directly in front of the antennas at a height within their field of view. The red curves mark the ground-truth contours of two small $(\mathrm{D}=16 \mathrm{~cm})$ and large $(\mathrm{D}=40 \mathrm{~cm})$ cylinders, centered at $2.3 \mathrm{~m}$ and $3.6 \mathrm{~m}$ respectively, and two small $(\mathrm{L}=30 \mathrm{~cm})$ and medium $(\mathrm{L}=48 \mathrm{~cm})$ plates, the latter on a $30^{\circ}$ slant, placed at $4.6 \mathrm{~m}$ and $5.7 \mathrm{~m}$. The medium plate appears more faint in the image than the small plate, despite its closer range and size, because the slant deflects the arrivals from the receiver, but its shape is nevertheless distinct. This experiment demonstrates the ability of the mobile robot to clearly discriminate with high resolution the irradiated contours of the reflectors in a scene, even at long range. The apparent noise in the image is actually due to reflections from the lights and beams of the removable ceiling. Note the sheetrock backwall at $6 \mathrm{~m}$ from the scan plane: reflectors at the center of the scan appear within the real beamwidth of the antennas at most positions during the scan, generating the greatest total receiver power from the object such that it appears strongest in the image; this explains why the aluminum studs within the backwall are visible while those at the far ends lie below the dynamic range of the system. Rather in Fig. 3(b), a 3-meter scan of same wall type taken at the close range of $0.75 \mathrm{~m}$ clearly reveals the regular spacing of the stud clusters every 16" $(40.6 \mathrm{~cm})$ as well as the two layers of the sheetrock wall. The small and large plates are also visible at $1.6 \mathrm{~m}$ and $2.3 \mathrm{~m}$ respectively.

The segment displayed in Fig. 3(b) actually makes up the bump-in of the extended wall shown in Fig. 3(c) captured from a much longer 9-meter scan, however from about 3 meters away; in fact, the identical stud pattern can be recognized indexed as 5 to 8 meters on the cross-range. Note the small cylinder centered at $2.2 \mathrm{~m}$ in line-of-sight as well as the reflectors placed on the other side of the wall: the side of a thin bookcase $(\mathrm{L}=20 \mathrm{~cm})$ and the small plate both at 3.7 $\mathrm{m}$, the medium plate at $5.4 \mathrm{~m}$, and a large plate $(\mathrm{L}=85 \mathrm{~cm})$ at $7 \mathrm{~m}$. The scan also reveals the presence of two metal ducts $(\mathrm{L}=30 \mathrm{~cm})$ inside the left side of the wall. The wall seems to bend as the studs stray from the ground-truth red curve most noticeably after $6 \mathrm{~m}$ on the cross-range. This stems from the finite tolerance of the robot wheel axle which deviates the scan from a perfect straight line. The deviation is only observable in long scans such as this.

Fig. 3(d) displays the radar image of a plaster wall with wooden studs generated from a 6-meter scan. Plaster is more reflective than sheetrock and so the wall is seen more clearly in this image, and as expected the dielectric studs are not visible at all. The small cylinder is centered at $1.9 \mathrm{~m}$ in lineof-sight while the large cylinder is centered behind the wall at $3.9 \mathrm{~m}$ along with the small and medium plates at $3.5 \mathrm{~m}$ and the large plate at $5.5 \mathrm{~m}$. As applies to the slanted plate in Fig. 3(a), cylinders deflect the arrivals from the receiver, and so the contour of the large cylinder seems faint with respect to the medium plate roughly the same size and at roughly the same range.

\section{CONCLUSIONS}

In this work, we have designed and built a novel UltraWideband radar system with synthetic aperture realized through a variable-length scanner or mobile robot. The wide dynamic range of the system coupled with its unrestricted aperture length allows us to generate high-resolution throughthe-wall images up to a range of 8 meters. The results are substantiated through a set of images generated from measurements taken in different environments through sheetrock walls with aluminum studs and plaster walls with wooden studs. The images clearly show the wall detail including stud placement as well as the contours of circular and flat objects both in the foreground and background. 


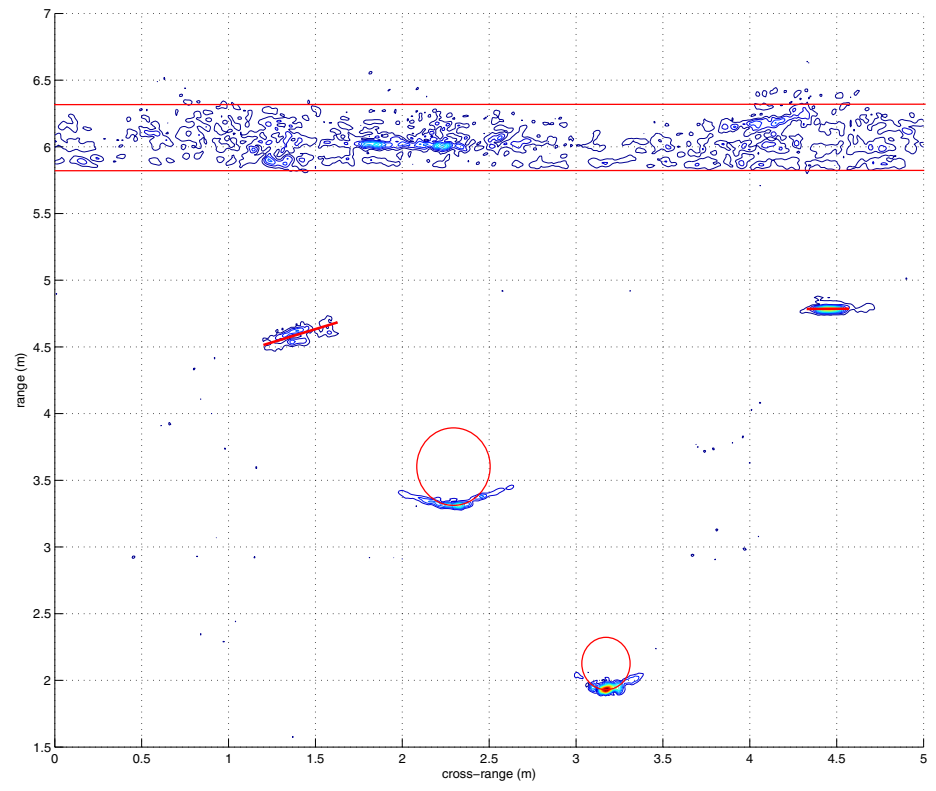

(a) Reflectors in line-of-sight and wall in background.

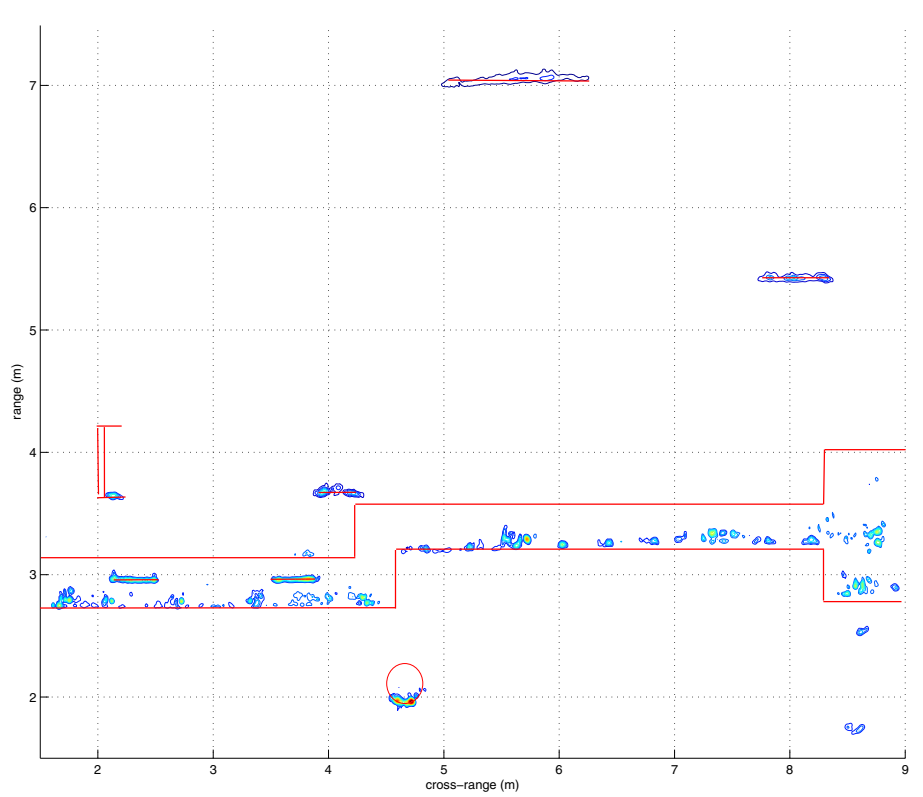

c) 9-meter scan of sheetrock wall with aluminum studs showing wall bump-in and reflectors on both sides of wall.

Fig. 3. Images generated from our UWB radar system.

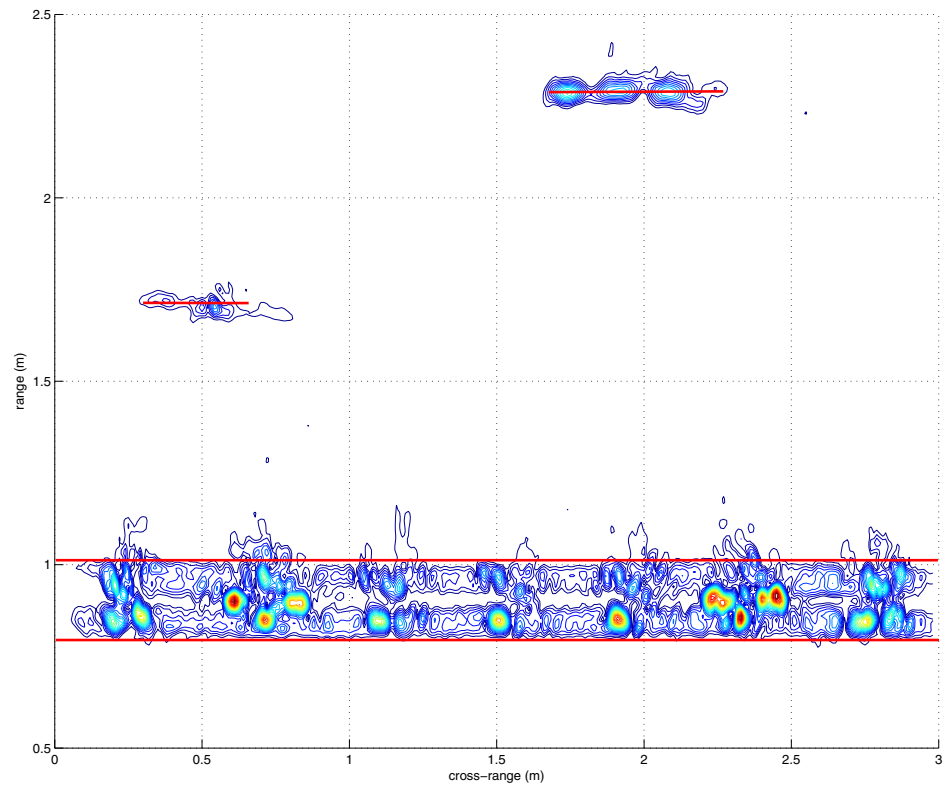

(b) Close-wall scan showing aluminum studs with two plate reflectors on opposite side of wall.

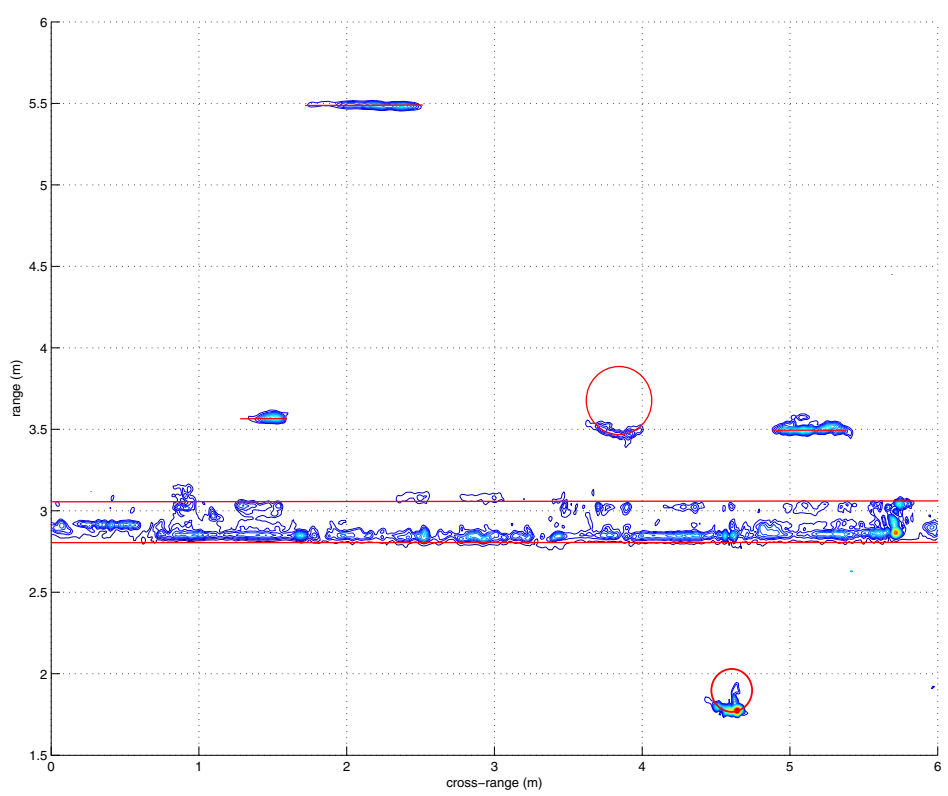

(d) Plaster wall with wooden studs with reflectors on both sides of wall. 


\section{REFERENCES}

[1] V.M. Lubecke, O. Boric-Lubecke, A. Host-Madson, and A.E. Fathy, "Through-the-Wall Radar Life Detection and Monitoring," IEEE Microwave Symposium, pp. 769-772, June 2007.

[2] M. Mahfouz, A. Fathy, Y. Yang, E. Elhak Ali, and A. Badawi, "Seethrough-Wall Imaging using Ultra Wideband Pulse Systems," IEEE Applied Imagery and Pattern Recognition Workshop, pp. 19-21, Oct. 2005.

[3] M. Lin, Z. Zhongzhao, and T. Xuezhi, "A Novel Through-Wall Imaging Method using Ultra WideBand Pulse System," IEEE Conf. on Intelligent Hiding and Multimedia Signal Processing, pp. 147-150, Dec. 2006.

[4] C. Gentile and A. Kik, "A Comprehensive Evaluation of Indoor Ranging Using Ultra-Wideband Technology," EURASIP Journal on Wireless Communications and Networking, vol. 2007, id. 86031, 2007.

[5] F.-C. Chen and W.C. Chew, "Time-Domain Ultra-Wideband Microwave Imaging Radar System," IEEE Instrumentation and Measurement Technology Conf., pp. 648-650, May 1998.

[6] F. Ahmad, M.G. Amin, and S.A. Kassam, "Synthetic Aperture Beamformer for Imaging through a Dielectric Wall," IEEE Trans. on Aerospace and Electronic Systems, vol. 41, no. 1, pp. 271-283, Jan. 2005.

[7] F. Ahmad, M.G. Amin, and S.A. Kasaam, "A Beamforming Approach to Stepped-Frequency Synthetic Aperture Through-the-Wall Radar Imaging," IEEE Workshop on Computational Advances in Multi-Sensor Adaptive Processing, pp. 24-27, Dec. 2005.

[8] F. Ahmad and M.G. Amin, "Through-the-Wall Radar Imaging Experiments," IEEE Workshop on Signal Processing Applications for Public Safety and Forensics, pp. 1-5, April 2007.

[9] S. Hantscher, A. Reisenzahn, and C.G. Diskus, "An UWB Wall Scanner Based on a Shape Estimating SAR Algorithm," IEEE/MTT-S Microwave Symposium, pp. 1463-1466, June 2007.

[10] S. Hantscher, B. Praher, A. Reisenzahm, and C.G. Diskus, "2D Imaging Algorithm for the Evaluation of UWB B-Scans," IEEE Conf. on Ultrawideband and Ultrashort Impulse Signals, pp. 139-141, Sept. 2006.

[11] I. Nicolaescu, P. Van Genderen, K.W. Van Dongen, J. Van Heijenoort, and P. Haakaart, "Stepped Frequency Continuous Wave Radar-Data Preprocessing," Workshop on Advanced Ground Penetrating Radar, pp. 177-182, May 2003.

[12] A.G. Yaravoy, "UWB Array-Based Sensor for Near-Field Imaging," IEEE Trans. on Microwave Theory and Techniques, vol. 55, no. 6, June 2007.

[13] E. Yigit, S. Demirci, C. Ozdemir, and A. Kavak, "A Synthetic Aperture Radar-Based Focusing Algorithm for B-Scan Ground Penetrating Radar Imagery," Microwave and Optical Technology Letters, vol. 29, no. 10, Oct. 2007.

[14] Y. Yang and A. Fathy, "Design and Implementation of a Low-Cost RealTime Ultra-Wide Band See-Through-Wall Imaging Radar System," IEEE Microwave Symposium, pp. 1467-1470, June 2007.

[15] W.G. Carrara, R.S. Goodman, and R.M. Majewski, "Spotlight Synthetic Aperture Radar," Artech House, Inc., 1995.

[16] H. Hashemi, "The Indoor Radio Propagation Channel," Proceedings of the IEEE, vol. 81, no. 7, pp. 943-968.

[17] X. Li. and K. Pahlavan, "Super-Resolution TOA Estimation With Diversity for Indoor Geolocation," IEEE Trans. on Wireless Communications, vol. 3, no. 1, Jan. 2004.

[18] J. Keignart and N. Daniele, "Subnanosecond UWB Channel Sounding in Frequency and Temporal Domain," IEEE Conf. on Ultra Wideband Systems and Technologies, pp. 25-30, May 2002.

[19] J. Gazdag and P. Sguazzero, "Migration of Seismic Data," Proceedings of the IEEE, vol. 72, no. 10, pp. 1302-1315, Oct. 1994

[20] D.J. Daniels "Ground Penetrating Radar, Second Edition," IEE Radar, Sonar and Navigation, Series 15, 2004. 